\title{
Metal-Organic Frameworks for Immobilizing Enzymes: Optimization and Improvement
}

\author{
Chao Zhong, Guorong Li, and Zian Lin* \\ Ministry of Education Key Laboratory of Analytical Science for Food Safety and Biology, Fujian Provincial Key \\ Laboratory of Analysis and Detection Technology for Food Safety, College of Chemistry, \\ Fuzhou University, Fuzhou, Fujian 350116, China
}

E-mail: zianlin@fzu.edu.cn (Z. L.)

\begin{abstract}
Enzymes are biomolecules with remarkable catalytic properties, which play a critical role in the driving toward green and sustainable methodologies for chemicals manufacturing. In spite of many advantages in enzyme catalysis, it remains fragile entities. These defects limit the widespread application of enzymes in industry. It is a favorable choice to immobilize the enzyme on solid supports to enhance its activity and stability. Metal-organic frameworks (MOFs) are excellent porous materials that possess tunable porosity, desirable functionality, extremely high surface area, and excellent chemical/thermal stability. It has made great achievements in the encapsulation of enzymes in the past few years. In this article, we focus on the recent advances of these composites, especially in the encapsulated strategies and mechanism, and future perspectives are discussed as well
\end{abstract}

Keywords metal-organic frameworks, enzyme, nanomaterials, immobilization

The construction of biocomposite nanomaterials, where biomolecules are encapsulated in nanostructured materials, remains a great challenging but promising area of research, due to the improved anti-denaturation, activity and even selectivity of the immobilized biomolecules. As an application branch of biocomposites, immobilized enzyme reactors attract great interest, where the immobilized enzymes have higher catalytic activity and stability, compared with free enzymes. In the past few years, various solid supports, including nano/microgels, ${ }^{[1,2]}$ hollow $^{[3]}$ and organic-inorganic hybrid nanoflowers, ${ }^{[4]}$ have been explored to immobilize enzymes by chemical covalent bonding, trapping or physically adsorption. However, there remains great challenge such as decreased accessibility of enzyme active sites, enhanced restriction of matrix on enzymatic reaction mass transfer, and denaturation of enzyme, in the process of enzyme immobilization. Therefore, developing the effective strategy and exploring new nanomaterials may be an available way to solve the above problems.

Metal-organic frameworks (MOFs) are a type of porous material that has tunable porosity, desirable functionality, extremely high surface area, and excellent chemical/thermal stability. Owing to the unique properties of MOFs, it has drawn considerable attention as a potential solid support for enzyme immobilization. However, since the size of the protein molecules is generally larger than the pore size of MOFs, conventional methods for the preparation of enzyme-embedded MOFs was a great challenge. Inspired by the biological mechanisms involved in protein transport through membranes, Chen et al. ${ }^{[5]}$ demonstrated for the first time that Cytochrome $C$ (Cyt c) could enter the interior cavities of MOFs that the pore size of MOFs was small relative to the molecular dimension of Cyt c. The results of fluorescence studies indicated that Cyt c underwent conformational changes during the immobilization process, which was different from its native conformation and denatured conformation. ${ }^{[5]}$ This work opens up a new field for employing MOFs as a platform for enzyme immobilization. Nevertheless, this aforementioned method is limited in further application due to high requirements for the nature of enzymes. Therefore, exploring more mildly and widely available immobilization methods, where synthetic process should occur under approximate physiological condition so as to guarantee that the enzymes remain stably bioactive, are highly desirable. Among all the reported MOFs, ZIF- $8^{[6]}$ has aroused great interest due to its exceptional chemical and thermal stability and negligible cytotoxicity. As a result, two efficient strategies have been developed for the synthesis of such composites: controlled coprecipitation using polyvinylpyrrolidone (PVP) and biomimetic mineralisation.

In a unique and innovative work, Lyu et al. ${ }^{[7]}$ proposed a controlled coprecipitation strategy in which a solution containing both Cyt $c$ and PVP was directly mixed with a methanol solution of zinc nitrate hexahydrate and 2-methylimidazole to synthesize Cyt c@ZIF-8 composites at room temperature, where the PVP played a key role in assisting the dispersion and stabilization of Cyt $\mathrm{c}$ in methanol. ${ }^{[7]}$ The resultant composites showed a 10 -fold increase in the peroxidase activity compared to the free Cyt $\mathrm{c}$ in solution. The enhancement of enzyme activity in Cyt c@ZIF-8 was mainly attributed to the following two factors. ${ }^{[8]}$ On one hand, the substrate affinity toward $\mathrm{H}_{2} \mathrm{O}_{2}$ increased due to the incubation of Cyt $\mathrm{c}$ in methanol, which led to a conformational change of Cyt $c$ to expose its heme group. On the other hand, the interaction between the embedded Cyt $\mathrm{c}$ and $\mathrm{Zn}^{2+}$ in $\mathrm{ZIF}-8$ crystals could enhance the catalytic activity of Cyt c. ${ }^{[7]}$ In addition, Liang et al. ${ }^{[8]}$ extended biomimetic mineralization from inorganic minerals to MOFs immobilized enzymes. The results validated enzyme could effectively induce MOFs to form high activity and stability biocomposites under physiological conditions without PVP. ${ }^{[9]}$ This rapid, low-cost biomimetic mineralization process gives rise to new possibilities for the exploitation of biomacromolecules.

Although great achievements have been made in the 


\section{Perspective}

encapsulation of enzymes, the methods to improve the activity of enzymes in composites and to fully utilize the functions of MOFs still need to be explored. Shieh et al. ${ }^{[10]}$ demonstrated new functions for biocatalysts by embedding catalase molecules into ZIF-90. A solution containing both catalase (CAT) and capping agent was directly mixed with an aqueous solution of zinc nitrate and imidazolate-2-carboxaldehyde to synthesize CAT@ZIF-90 composites, which retained the peroxidase activity at room temperature. ${ }^{[9]}$ The ZIF-90 support provided an interesting size-sheltering function to catalase and protected catalase from the inhibitor proteinase $\mathrm{K}^{\left[{ }^{[9]}\right.}$ This work provides a new perspective, that is to say, the pores of MOFs can act as a barrier to block the denatured factors of the enzyme and increase the resistance of the enzyme to undesirable environments. ${ }^{[11]}$ Recently, Pan et al. ${ }^{[10]}$ demonstrated for the first time the characterization of the orientation of enzymes trapped on the ZIF-8 surface. This work demonstrated the possibility to enable substrate selectivity based on the size of support and affinity to MOFs. ${ }^{[10]}$ Moreover, Liang et al. ${ }^{[11]}$ also showed that enzymes encapsulated within hydrophilic MAF-7 or ZIF-90 retains enzymatic activity when exposed to high temperatures, denaturing or proteolytic agents, and organic solvents, whereas hydrophobic ZIF-8 afforded negligible protection to urease and catalase (Figure 1). It was indicated that optimizing hydrophobic/hydrophilic interactions between enzymes and MOFs was essential for the stability of biomolecules. ${ }^{[11]}$

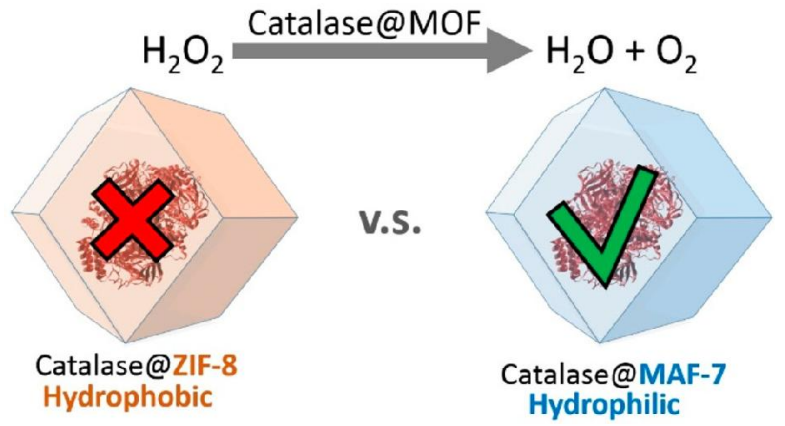

Figure 1 Activity of enzymes influenced by hydrophilicity/ hydrophobicity of MOF. Reproduced with permission from Ref. [11]. Copyright 2019, American Chemical Society.
In conclusion, we present a comprehensive review on the recent advances in enzyme@MOFs composites. As an excellent solid support, MOFs has been applied in enzyme immobilization successfully. However, there are still some problems to be resolved, such as an insignificant increase in the activity of the enzyme, and the limitation in the type of MOFs applied to enzyme immobilization. Therefore, it is necessary to further explore the mechanism of the enzyme orientation during the immobilization process and the influence of the MOFs structure on the enzyme activity.

\section{References}

[1] Yan, M.; Ge, J.; Liu, Z.; Ouyang, P. J. Am. Chem. Soc. 2006, 128, 11008.

[2] Schachschal, S.; Adler, H.-J.; Pich, A.; Wetzel, S.; Matura, A.; van Pee, K.-H. Colloid Polym. Sci. 2011, 289, 693.

[3] Ji, X.; Wang, P.; Su, Z.; Ma, G.; Zhang, S. J. Mater. Chem. B 2014, 2, 181.

[4] Ge, J.; Lei, J.; Zare, R. N. Nat. Nanotechnol. 2012, 7, 428.

[5] Chen, Y.; Lykourinou, V.; Vetromile, C.; Hoang, T.; Ming, L. J.; Larsen, R. W.; Ma, S. J. Am. Chem. Soc. 2012, 134, 13188.

[6] Hayashi, H.; Cote, A. P.; Furukawa, H.; O’Keeffe, M.; Yaghi, O. M. Nat. Mater. 2007, 6, 501.

[7] Lyu, F.; Zhang, Y.; Zare, R. N.; Ge, J.; Liu, Z. Nano. Lett. 2014, 14, 5761.

[8] Liang, K.; Ricco, R.; Doherty, C. M.; Styles, M. J.; Bell, S.; Kirby, N.; Mudie, S.; Haylock, D.; Hill, A. J.; Doonan, C. J.; Falcaro, P. Nat. Commun. 2015, 6, 7240.

[9] Shieh, F. K.; Wang, S. C.; Yen, C. I.; Wu, C. C.; Dutta, S.; Chou, L. Y.; Morabito, J. V.; Hu, P.; Hsu, M. H.; Wu, K. C.; Tsung, C. K. J. Am. Chem. Soc. 2015, 137, 4276.

[10] Pan, Y.; Li, H.; Farmakes, J.; Xiao, F.; Chen, B.; Ma, S.; Yang, Z. J. Am. Chem. Soc. 2018, 140, 16032.

[11] Liang, W.; Xu, H.; Carraro, F.; Maddigan, N. K.; Li, Q.; Bell, S. G.; Huang, D. M.; Tarzia, A.; Solomon, M. B.; Amenitsch, H.; Vaccari, L.; Sumby, C. J.; Falcaro, P.; Doonan, C. J. J. Am. Chem. Soc. 2019, 141, 2348. 